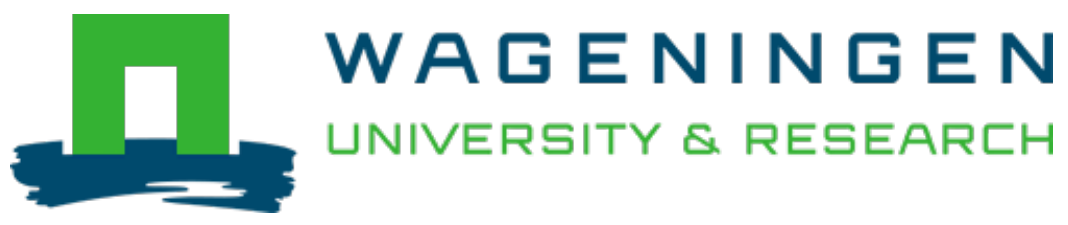

\title{
Predictability of Biotic Stress Structures Plant Defence Evolution
}

\author{
Trends in Ecology and Evolution
}

Mertens, Daan; Boege, Karina; Kessler, André; Koricheva, Julia; Thaler, Jennifer S. et al

https://doi.org/10.1016/j.tree.2020.12.009

This article is made publicly available in the institutional repository of Wageningen University and Research, under the terms of article $25 \mathrm{fa}$ of the Dutch Copyright Act, also known as the Amendment Taverne. This has been done with explicit consent by the author.

Article $25 \mathrm{fa}$ states that the author of a short scientific work funded either wholly or partially by Dutch public funds is entitled to make that work publicly available for no consideration following a reasonable period of time after the work was first published, provided that clear reference is made to the source of the first publication of the work.

This publication is distributed under The Association of Universities in the Netherlands (VSNU) 'Article $25 \mathrm{fa}$ implementation' project. In this project research outputs of researchers employed by Dutch Universities that comply with the legal requirements of Article $25 \mathrm{fa}$ of the Dutch Copyright Act are distributed online and free of cost or other barriers in institutional repositories. Research outputs are distributed six months after their first online publication in the original published version and with proper attribution to the source of the original publication.

You are permitted to download and use the publication for personal purposes. All rights remain with the author(s) and / or copyright owner(s) of this work. Any use of the publication or parts of it other than authorised under article $25 \mathrm{fa}$ of the Dutch Copyright act is prohibited. Wageningen University \& Research and the author(s) of this publication shall not be held responsible or liable for any damages resulting from your (re)use of this publication.

For questions regarding the public availability of this article please contact openscience.library@wur.nl 


\title{
Trends in Ecology \& Evolution
}

\section{Predictability of Biotic Stress Structures Plant Defence Evolution}

\author{
Daan Mertens, ${ }^{1, @, \star ~ K a r i n a ~ B o e g e, ~}{ }^{2, @}$ André Kessler, ${ }^{3}$ Julia Koricheva, ${ }^{4, @ ~ J e n n i f e r ~ S . ~ T h a l e r, ~}{ }^{5, @ ~}$ \\ Noah K. Whiteman, ${ }^{6}$ and Erik H. Poelman ${ }^{1, \star}$
}

To achieve ecological and reproductive success, plants need to mitigate a multitude of stressors. The stressors encountered by plants are highly dynamic but typically vary predictably due to seasonality or correlations among stressors. As plants face physiological and ecological constraints in responses to stress, it can be beneficial for plants to evolve the ability to incorporate predictable patterns of stress in their life histories. Here, we discuss how plants predict adverse conditions, which plant strategies integrate predictability of biotic stress, and how such strategies can evolve. We propose that plants commonly optimise responses to correlated sequences or combinations of herbivores and pathogens, and that the predictability of these patterns is a key factor governing plant strategies in dynamic environments.

\section{Plants in a Variable and Multistressor Environment}

Individual organisms use historical and current information to adapt their physiology and behaviour to retain fitness under forthcoming conditions [1,2]. For sessile organisms that cannot evade suboptimal environments by moving, fitness is strongly determined by phenotypic responses to changes in (a)biotic conditions. Plants serve as ideal model systems for understanding how sessile organisms use information and adapt their phenotype in a community context [3]. Over their lifetimes, plants cope with a range of stresses, such as daily changes in insolation and temperature, low resource availability, unfavourable weather, competition, and attack by pathogens or herbivores [4,5]. In many cases, stressors are immediate and long lasting [6]. However, probability of occurrence of biotic stressors as well as the sequence and combination in which they occur can be highly variable [7]. Consequently, plants have evolved phenotypic plasticity to fine-tune their responses to the multiple stressors present in the environment in which they develop and persist $[5,8,9]$.

Most of our research on plant plasticity has focussed on how plants maximise resilience to current stress. However, more recently, plant physiological and ecological studies have highlighted how plant responses to current stress not only affect plasticity in response to future stress $[10,11]$ but also how this plasticity alters the likelihood of future stress [12,13]. Importantly, even though stressors may vary greatly across and within seasons and over the life cycle of plants, their occurrence is not random. Foreseeable patterns may emerge from the phenology of insect herbivores and activity patterns of vertebrate herbivores associated with optimal abiotic conditions [14], the responses of herbivores and pathogens to specific plant (ontogenetic) phenotypes [15], or the increased likelihood of simultaneous or sequential attacks by different plant antagonists [13]. For example, open wounds in plant tissues caused by chewing herbivores increase the risk of pathogen attack [16]. Such correlations provide plants with an opportunity to adjust their phenotype in anticipation of suboptimal conditions or dynamics of stress. Even though it has been well established that anticipatory responses (see Glossary) in plants are ubiquitous [17], we have little understanding of the ecological conditions that promote the evolution of anticipatory

\section{Highlights}

Sessile organisms such as plants strongly rely on external stimuli that predict the onset of stress to anticipate suboptimal conditions and adjust their phenotype accordingly.

By integrating correlations among attackers as well as predictable patterns in the timing, and order of arrival of attack in their defence strategies, plants can anticipate biotic stress and maximise life-time fitness. However, it is unclear under which ecological conditions such strategies are adaptive.

Selection on plants to integrate predictable patterns of future stressors in their strategies when responding to current stress can lead to suboptimal responses to each stressor in isolation. Explicitly including the predictability of cooccurrence and temporal patterns of stressors in theory on plant defence strategies is crucial in understanding the evolution of plant growth-defence and reproductive strategies.
${ }^{1}$ Laboratory of Entomology, Wageningen University and Research, P.O. Box 16, 6700 AA, Wageningen, The Netherlands ${ }^{2}$ Instituto de Ecología, Universidad Nacional Autónoma de México, Ciudad Universitaria, Apartado Postal 70-275, Coyoacán, C.P. 04510 , Ciudad de México, Mexico ${ }^{3}$ Department of Ecology and Evolutionary Biology, Cornell University, Ithaca, NY 14853, USA ${ }^{4}$ Department of Biological Sciences, Royal Holloway University of London, Egham, Surrey TW20 0EX, UK ${ }^{5}$ Department of Entomology, Cornell University, Ithaca, NY 14853, USA ${ }^{6}$ Department of Integrative Biology, University of California-Berkeley, Berkeley, CA 94720, USA 
strategies, especially in relation to biotic stress, or under which conditions such strategies are adaptive [18]. Where most theory on plant growth-defence strategies and their evolution thus revolves around responses to current stress, predicting and anticipating forthcoming stress may be an adaptive strategy under various ecological scenarios [19] and should be prominently included in our theories on plant plasticity.

Plants are likely to commonly optimise responses to correlated sequences or combinations of herbivores and pathogens, rather than optimising responses to current stress. We apply the concept of ecological forecast horizons, developed to quantify the accuracy of predictions on community and ecosystem processes, to plant defence strategies [20,21]. We define a plant's forecast horizon as the timespan measured from the (static) point in time at which the plant has obtained information for which a plant can make more accurate predictions on, and hence prepare for, forthcoming stress conditions than when following nonanticipatory strategies. Plant defence strategies incorporate a forecast horizon to cope with attacks by herbivores and pathogens following a gradient of predictability ranging from wholly unpredictable to highly predictable. Anticipatory strategies fundamentally revolve around a cost-benefit balance [22], but existing theory can be critically expanded by explicitly incorporating the predictability of biotic stress in risk management strategies.

\section{Using information from the Environment to Predict Future Stress}

The forecast horizon of plants is determined by their ability to obtain information that correlates with future environmental and ecological conditions [23]. The process of information gathering in plants and the subsequent responses to stress involve numerous sensory mechanisms and have been thoroughly reviewed [17] (Table 1). As most stressors persist over long periods of time, current conditions experienced by plants are likely to correlate well with the environment that plants will experience in the near future. A simple example is the daily variation in light levels caused by the Earth's rotation on its axis (night vs day). Circadian rhythms have evolved in response to this predictable diurnal flux in light (and temperature) levels [24]. An example of a similar evolutionary response to biotic stress is that some plants can detect oviposition by phytophagous insects, which correlates well with likely future herbivory by larvae hatching from these eggs [1]. Plants that prime or induce defences in response to eggs gain a fitness advantage over those plants without the ability to use this cue [16].

In addition to a direct interaction with specific stressors, plants can obtain information from more indirect cues or from the correlations between different stressors [25]. As attacker communities are structured over time because of differences in phenology, life history, and niche differentiation, interactions with specific attackers can become reliable predictors of future biotic stress by different attackers. For example, leaf shelters constructed by caterpillars of the genus Pseudotelphusa on white oak Quercus alba increase the species richness of subsequent herbivores that colonize the new niches provided by these leaf shelters [12]. In addition to niche construction, herbivore-induced changes in plants can generate plant-mediated interaction linkages between herbivores. Plant responses to an initial attacker can thus affect the likelihood of colonisation by subsequent herbivores $[13,26]$. These correlations among antagonists can be immediate such as found for the correlated attack for pathogens spread by insect vectors [27], or spanning over longer periods of time and even across seasons for perennial plants, such as found for the correlations between transient attack by herbivores and their legacy effects on future biotic stress [28]. Thus, by perceiving the current herbivore interaction as a cue for the type of future attack, plants may incorporate likely future stress in their response to the current attacker.

\author{
${ }^{*}$ Correspondence: \\ daan.mertens@wur.nl (D. Mertens) and \\ erik.poelman@wur.nl (E.H. Poelman). \\ ${ }^{\circledR}$ Twitter: @daan_mertens (D. Mertens), \\ @karinaboege (K. Boege), \\ @KorichevaLab (J. Koricheva), and \\ @ToxicPlantLab (J.S. Thaler).
}


Table 1. Overview of Different Plant States or Stimuli That Plants Respond to, Which May Correlate with Dynamics in Future Stress Conditions

\begin{tabular}{|c|c|c|}
\hline Plant state or stimulus & Example & Refs \\
\hline \multicolumn{3}{|l|}{ Plant intrinsic state } \\
\hline \multicolumn{3}{|l|}{ Intragenerational } \\
\hline Architecture & $\begin{array}{l}\text { Apical stem bending alters susceptibility to aphids and gall-forming } \\
\text { herbivores in late goldenrod (Solidago altissima) }\end{array}$ & [30] \\
\hline Biomass & $\begin{array}{l}\text { Leaf biomass determines leaf-chewing insect abundance across } \\
\text { tropical forest host species }\end{array}$ & [70] \\
\hline Chemical composition & $\begin{array}{l}\text { Insect community structure covaries with host plant chemistry in } \\
\text { pedunculate oak (Quercus robur) }\end{array}$ & [71] \\
\hline Ontogenetic state & Floral volatiles attract specialist herbivores in the Cucurbita genus & [72] \\
\hline \multicolumn{3}{|l|}{ Transgenerational } \\
\hline Seed composition & $\begin{array}{l}\text { Parental stress directly affects the metabolome of seeds in thale cress } \\
\text { (Arabidopsis thaliana) }\end{array}$ & [73] \\
\hline Seed germination & $\begin{array}{l}\text { Regulation of seed dormancy following maternal herbivory in } \\
\text { Arabidopsis thaliana }\end{array}$ & [74] \\
\hline Trait plasticity & $\begin{array}{l}\text { Abiotic stress enhances competitive ability of progeny in lady's } \\
\text { thumb (Polygonum persicaria) }\end{array}$ & [75] \\
\hline
\end{tabular}

\section{External cues or stressors}

Abiotic

Photoperiod

Salinity

Touch

Vibrations

Wind

Gravitropism

Humidity gradients

Chemicals in

atmosphere

Chemicals in soil
Light quality affects flavonoid production in wheel wingnut (Cyclocarya paliurus)

Altered photoperiod induces stress in Arabidopsis thaliana

Heat stress alters shock-factor-responsive gene expression in Arabidopsis thaliana

Rapid and gradual decreases in temperature trigger different pathways in Arabidopsis thaliana

Fire induced heat alleviates dormancy across grassland species in South-Eastern Australia

Salinity reduces the capacity of the photosynthetic system in cabbage (Brassica oleracea)

Rapid leaflet-closure responses to mechanical stimulation in Mimosa pudica

Obstacle avoidance by self-inhibition in pea (Pisum sativum)

Sound vibrations increase expression of genes related to mechanical stimulation in Arabidopsis thaliana

Air flow increases height, while stem flexure reduces height in common sunflowers (Helianthus annuus)

Gravity reception is used to regulate organ straightening and

Hydrotropism is regulated by auxin and abscisic acid in Arabidopsis thaliana

Ozone modifies plant responses to biotic stresses in charlock mustard (Sinapis arvensis)

Smoke and ash induce germination across functional groups in Northern European heathlands

Root tip contact with low-phosphate media reprograms plant root architecture in Arabidopsis thaliana plant posture in Arabidopsis thaliana

\section{Glossary}

Anticipatory responses: a plant response to information indicative of stress in which the phenotype is adjusted in anticipation of suboptimal conditions or arrival of stress.

Forecast horizon: the maximal length of time into the future from the point in time at which information is gathered for which plants can make predictions about, and hence anticipate, forthcoming stress conditions. Induced response: the phenotypic change of a plant in response to stress that may lead to decreased performance of the stressor (induced resistance) and result in a plant fitness benefit by the response (induced defence). Intrinsic predictability: the maximal degree of statistical predictability among successive environmental states within a local environment.

Phenological and/or conditional state of the plant: the intrinsic state of the plant determined by ontogenetic and physiological phenotype formed by prior interactions with the (a)biotic environment.

Plant-mediated interaction: the indirect effects of spatially or temporally separated organisms on each other's performance or behaviour through induced responses of their shared host plant.

Predictability of stress: the level of certainty in arrival patterns of stress such as the timing and order of arrival of herbivore or pathogen species over a plant's lifetime.

Priming: the phenomenon whereby a temporally limited environmental (priming) stimulus prepares and modifies the response to a future stress incident (the triggering stimulus). 


\begin{tabular}{|c|c|c|}
\hline Plant state or stimulus & Example & Refs \\
\hline \multicolumn{3}{|l|}{ Biotic } \\
\hline Mycelia & $\begin{array}{l}\text { Common mycelial networks warn neighbouring plants of aphid } \\
\text { attack in faba bean (Vicia faba) }\end{array}$ & [90] \\
\hline Bioacoustics & $\begin{array}{l}\text { Flowers respond to pollinator sound by increasing nectar sugar concentration } \\
\text { in the evening primrose (Oenothera drummondii) }\end{array}$ & {$[91]$} \\
\hline \multirow[t]{2}{*}{ Volatiles } & $\begin{array}{l}\text { Volatiles from damaged neighbours increase resistance against herbivores } \\
\text { across plant species }\end{array}$ & [92] \\
\hline & $\begin{array}{l}\text { The parasitic plant fiveangled dodder (Cuscuta pentagona) exhibits directed } \\
\text { growth toward volatiles of the potential plant host }\end{array}$ & [93] \\
\hline Nonvolatile exudates & $\begin{array}{l}\text { Root-secreted JA is involved in neighbour detection and plant-plant } \\
\text { communication in common wheat (Triticum aestivum) }\end{array}$ & [94] \\
\hline Herbivore damage & $\begin{array}{l}\text { Specific tobacco hawk moth (Manduca sexta) elicitor shows highly specialised } \\
\text { herbivore-detection system in solanaceous plants }\end{array}$ & [95] \\
\hline Vibrations & $\begin{array}{l}\text { Vibrations caused by insect feeding elicit chemical defences in Arabidopsis } \\
\text { thaliana }\end{array}$ & {$[96]$} \\
\hline \multirow[t]{2}{*}{ Herbivore oviposition } & $\begin{array}{l}\text { Oviposition by Leptinotarsa decemlineata beetles causes hypersensitivity and } \\
\text { egg drop in Solanum spp. }\end{array}$ & {$[97]$} \\
\hline & $\begin{array}{l}\text { Leaf contact cues associated with oviposition by the large cabbage white } \\
\text { (Pieris brassicae) arrest parasitoids in black mustard (Brassica nigra) }\end{array}$ & {$[98]$} \\
\hline \multirow[t]{2}{*}{ Herbivore excretion } & $\begin{array}{l}\text { Proteins from fall army worm (Spodoptera frugiperda) frass induces } \\
\text { wound-responsive defense genes in maize (Zea mays) }\end{array}$ & [99] \\
\hline & $\begin{array}{l}\text { Exogenous honeydew deposition by pea aphids Acyrthosiphon pisum alters } \\
\text { JA and SA accumulation in Vicia faba }\end{array}$ & {$[100]$} \\
\hline \multirow[t]{5}{*}{ Antagonist interactions } & $\begin{array}{l}\text { Barley yellow dwarf virus alters likelihood of herbivory by bird cherry-oat aphids } \\
\text { (Rhopalosiphum padi) in Triticum aestivum }\end{array}$ & {$[101]$} \\
\hline & $\begin{array}{l}\text { Herbivore damage increases attraction of generalist herbivores in creeping } \\
\text { cucumber (Solena amplexicaulis) }\end{array}$ & {$[102]$} \\
\hline & $\begin{array}{l}\text { Induced plant responses attract oviposition by the specialist small cabbage } \\
\text { whites (Pieris rapae) in radish (Raphanus raphanistrum) }\end{array}$ & {$[103]$} \\
\hline & $\begin{array}{l}\text { Order of herbivore arrival influences subsequent community development in } \\
\text { Brassica oleracea }\end{array}$ & {$[13]$} \\
\hline & $\begin{array}{l}\text { Infection with Pseudomonas spp. bacteria in leaves predicts prevalence and } \\
\text { damage by Scaptomyza flava }\end{array}$ & {$[48]$} \\
\hline
\end{tabular}

As the probability of interactions with a specific stressor is closely related to plant phenotype, the phenological and conditional state of the plant can be an important source of information that predicts to what type of stress it may get exposed. Moreover, transition between ontogenetic or phenological stages of plants such as germination, leaf flushing, or flowering are often initiated by similar abiotic conditions that trigger the release from dormancy in insects or migration of vertebrate herbivores. Hence, phenologies of plants and their attackers may strongly correlate [29]. The likelihood and impact of herbivore attack on plants may be strongly dependent on the plant phenotype expressed at each ontogenetic stage $[14,30]$ and on the ability of different ontogenetic stages of herbivores to cope with ontogenetic variation in plant phenotypes [31].

When stress conditions for parental plants closely correlate with conditions that are likely to be experienced by their offspring, it can be adaptive for parental plants to actively regulate ontogenetic trajectories or trait plasticity in the next generation $[32,33]$. Recent studies have highlighted the important role of epigenetic transgenerational mechanisms in the regulation of trait plasticity [34]. These mechanisms allow plants to alter the plasticity of offspring without requiring direct 
genetic variation, greatly increasing the responsiveness of plants to their environment over shorter trans-generational time scales (Box 1). Notably, such transgenerational plasticity fits well into a traditional evolutionary framework. The ability to alter the plasticity of a trait in offspring is likely a genetically encoded and heritable trait itself, resulting from Darwinian adaptation [35].

\section{Plants Evaluate and Integrate Information before Expressing Responses}

A significant problem for plants is that not all stimuli are relevant or can be used to anticipate future conditions in the plant's environment [23]. Identifying reliable cues from a background of environmental noise is thus a key challenge for plants using anticipatory defence strategies involving a forecast horizon. The first difficulty in the perception of information is that when the stimulus and the stress with which it correlates are separated over a wider timespan or longer distance, variation increases due to stochastic processes in the environment. Correlations between cues and the conditions they predict can rapidly deteriorate over spatial or temporal scales. This creates spatiotemporal variation in cue reliability, in which plants may be unable to correctly integrate or respond to the information they gather. A second challenge is that the correlations between single cues and stressors are often context-dependent; for example, the combination of the stressor with other cues. Exactly how accurate cues must be before they are considered reliable depends on the balance between the asymmetric fitness cost of errors. If the response threshold to a stimulus is too low, the costs of the defence response to a false alarm may outweigh the cost of being unprepared for attack $[22,36]$.

Possible solutions to these issues are, on the one hand, the integration of multiple cues in predictions, and, on the other hand, the selective weighting of more reliable cues over other by the plant [37]. It is clear that plants are able to respond conditionally to cues [17]. For example, repeated mechanical stimulation of leaflets by water droplets causes the sensitive plant, Mimosa pudica to decrease sensitivity in leaf closure to the point where leaflets no longer respond to the stimulus. After desensitisation to the stimulus, finger touch instead of water droplets elicited leaflet closure in the plants, thus excluding the possibility that desensitisation was due to fatigue [38].

\section{Box 1. Epigenetic Modulation of Plant Trait Plasticity across Generations}

Variation in most plant traits is quantitative (continuously variable) and the heritable component of this variation is polygenic in architecture - many genes each contribute a small effect to the phenotype. However, the expression of plant traits can be regulated by (heritable or transient) molecular mechanisms such as DNA methylation, small RNAs, or histone modifications affecting gene transcription. The epigenetic regulation of protein-coding genes is essential for general plant functions such as development or the silencing of transposable elements [104]. In addition to these general functions, epigenetic mechanisms play a key role in acclimation to stress and rapid plastic responses in plants [105]. Importantly, epigenetically controlled variation in trait plasticity exists without the need to deviate from a genetic blueprint - natural selection acts on the ability of a plant to epigenetically control trait expression - regulation of gene expression being a simple example $[106,107]$. Nongenetic inheritance through the transfer of epigenetic states can have major consequences on offspring resistance to (a)biotic stress [105]. These effects can be limited to one generation or persist for multiple generations by so called soft inheritance [108]. A critical need is to study the levels of biological organisation from individual to populations and species that produce these ontogenetically extended phenotypes. In addition, fully addressing this question requires identifying ultimate mechanisms at the genetical level that may drive the origin and persistence of transgenerational phenotypes.

Even though the mechanisms underlying epigenetic regulation of gene expression are becoming increasingly well studied, the effects of transgenerational phenotypic plasticity on plant responsiveness to environmental conditions and ultimately plant fitness are poorly understood. This is in great part due to complexity. A plant's phenotype is the result of interactions between its genotype, the inherited epigenetic elements, environmental conditions, within-generation epigenetic regulation, and other regulatory elements involved in plant development. Moreover, evidence suggests that nongenetically inherited elements that confer a fitness advantage are often and rapidly reversed, nuancing the implications of epimutations for long-term fitness of individuals. Despite its complexity, transgenerational phenotypic plasticity can be key to understanding the short-term integration of predictable patterns into plant life-history strategies. For example, information obtained by the maternal plant or both parental plants may correlate well with stress experienced by their progeny through spatial or temporal autocorrelation of stress. 
Opportunities and Challenges When Anticipating Future Conditions

When abiotic or biotic cues or stresses are sufficiently well correlated with future conditions that plants experience, it can be beneficial for plants to evolve anticipatory life-history strategies $[8,39,40]$. Anticipatory-induced responses to stimuli are ubiquitous in plants and can involve a primed state or actual phenotypic change in a wide range of physiological, chemical, or structural traits. These responses ultimately allow the plant to tolerate or avoid future stress conditions within its reaction norm (Box 2). The adaptiveness of an anticipatory response depends on opportunities and challenges in physiological regulation of the response, as well as on the overall predictability of the environment [20] (Figure 1).

Physiological Dilemmas in Plant Responses to Future Stress

Anticipatory strategies may be highly prevalent as they provide a solution to challenges plants are likely to face when responding to different attacks by herbivores and/or pathogens. First, even though early detection of stress by electrical signalling and reactive oxygen species (ROS) takes seconds to minutes, actual metabolic changes in the network of phytohormones and the formation of defence responses can take hours up to days to be realised [41,42]. This not only constrains plants in responding to current stress but also potentially makes the response suboptimal by the time other stressors arrive. Second, crosstalk between regulatory pathways may allow plants to fine-tune their responses to cope with simultaneous and sequential arrival of stressors $[43,44]$. However, it can also lead to a situation where the response to one stressor compromises an optimal response to another [10,43]. For example, activation of the salicylic acid (SA) pathway in response to attack by sap-sucking herbivores or biotrophic plant pathogens may impair jasmonic acid (JA)-based responses to chewing herbivores, and vice versa [43]. For anticipatory strategies to be optimal, plant responses should thus be effective against both the initial stress as well as the suite of future stressors the plant is likely to encounter. Natural selection should favour plant ability to integrate the correlated sequences of stressors in their physiological response to the initial attack, especially when the optimal response to the initial stressor would constrain responses to (more costly) future stressors. An emerging property is that plants may respond suboptimally to stressors in isolation to account for arrival of future stress this and is likely to contribute to the substantial variation found in plant responses to biotic stress [11].

\section{Box 2. Plant Anticipatory Strategies to Deal with (Un)predictable Stress}

In anticipatory strategies, plants adapt their phenotype in preparation of forthcoming adverse conditions. These strategies entail a range of genotypic and phenotypic regulations to match predictable arrival patterns of stress. The genetic regulation of anticipatory strategies is illustrated by ontogenetic trajectories of plants in expression of traits that are adaptive to stress. For example, seedlings that are heavily attacked by herbivores express stronger resistance to herbivory, because the costs of herbivory early in the development of plants are large. When plant biomass increases, the same degree of herbivore damage can be tolerated, and resources may be used for growth and reproduction. The adaptive value of ontogenetic changes in defence strategies match patterns that act on longer-term scales of development. Phenotypically, plants may use abiotic conditions to match seasonal variation in resistance to the likelihood of herbivore attack due to the correlation of seasonality and herbivore activity [29]. Over shorter timescales, plants may use reliable cues of presence of antagonists to anticipate actual attack by these antagonists. These cues may come from the environment, such as neighbouring plants that release volatiles when attacked by herbivores, or from direct interactions of the plant with stressors that predict forthcoming attack such as the oviposition of eggs by herbivorous insects on plant tissues $[1,109]$. Plants may anticipate the forthcoming stress by priming, a physiological process by which a plant prepares to respond to future biotic or abiotic stress more quickly or intensively, without the formation of resistance in the absence of the actual occurrence of stress. Alternatively, when the cue is highly reliable it pays plants to increase resistance through induced resistance [8]. The induced resistance may be tailored directly and primarily to the stressor associated with the cue, but we argue that these induced responses may also anticipate attack across longer temporal scales when the initial stressor is a good predictor for the arrival of other stressors. Across generations, plants may prepare offspring for forthcoming conditions by epigenetic mechanisms (Box 1). 


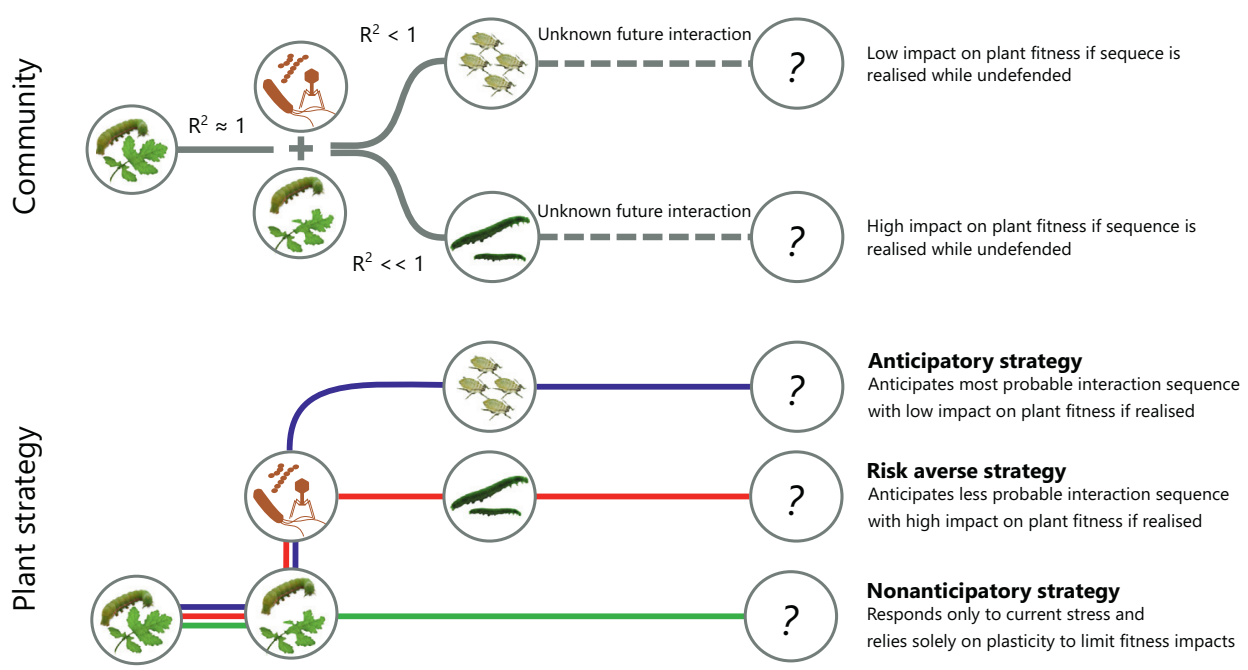

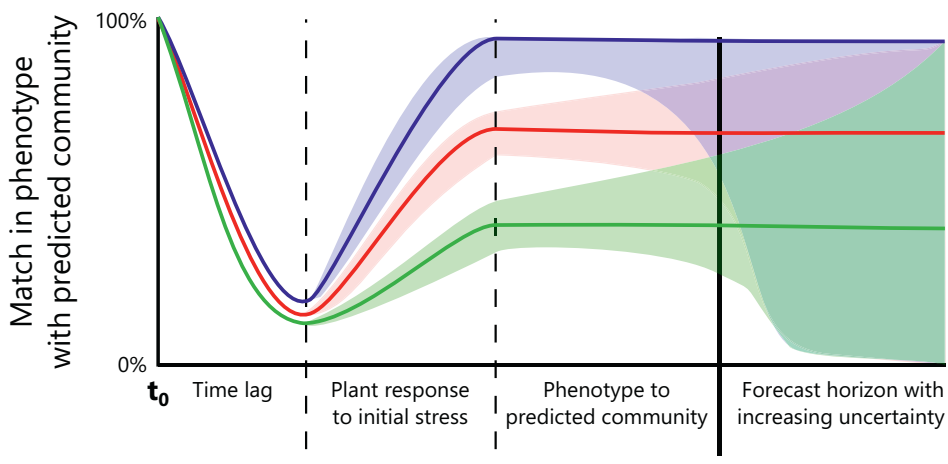

Time into future
Anticipatory strategy

Risk averse strategy

Nonanticipatory strategy

Trends in Ecology \& Evolution

Figure 1. Plant Strategies in the Framework of Forecast Horizons. Community: Plants interact with multiple biotic stressors in sequence. Stressors may strongly correlate (indicated by $\mathrm{R}^{2}$ ) such as when caterpillars cause open wounds that promote pathogen infections, or correlate only weakly such as illustrated by attack by a different herbivore species. Plant strategy: To deal with variation in predictability and the risk of incurring fitness costs, plants follow different strategies. The green line illustrates a trajectory in which the plant only responds to an initial stimulus and does not follow an anticipatory strategy. The red and blue lines illustrate trajectories with an anticipatory strategy, but in which plants differ in their proneness to take risk: The phenotypic trajectory illustrated by the blue line tracks the most probable antagonist community. The phenotypic trajectory illustrated by the red line is a risk averse strategy, where the plant anticipates a less likely, but if realized while unprepared, more costly scenario. Match in phenotype with the predicted community: Plant strategies vary in how well the plant phenotype will match with the predicted environment, represented by the coloured lines. The y axis represents how well the plant phenotype is predicted to match the future environment. From our vantage point at time zero (t0), we consider how well the plant will match the predicted communities of the future. Shaded areas indicate the increasing uncertainty in how well the phenotype will match the future community of antagonists due to stochastic processes, lag in community responses, and incorrect or incomplete information transferred by the initial attack.. Time lag: Plants need time to detect and start responding to the stimulus or stress, defined by physiological constraints. Plant response to initial stress: Time needed to fully form responses to the initial stress or stimulus. Anticipatory strategies match the changing antagonist community (red and blue lines), while non-anticipatory strategies only respond to the initial stressor or stimulus (green line). Developmental constraints and the integration of potential future stress in plant responses may limit how well plants following anticipatory strategies will match their phenotype with the environment. Phenotype to predicted community: Time frame where plants following anticipatory strategies try to match the future antagonistic community, and in which uncertainty increases until the forecast horizon is met, indicated by the solid black line. Forecast horizon with increasing uncertainty: In the period after the forecast horizon is met, predictions of plants following anticipatory strategies are not more accurate than noninformed/nonanticipatory strategies. 
Ecological Dilemmas in Plant Responses to Future Stress

How well plant responses match the actual conditions they anticipate and experience is highly dependent on the correlations between cues and stressors [45]. Even though dynamics in herbivore communities are often structured in their broad sense and offer plants the opportunity to anticipate stress, most plant interactions are characterised by high levels of stochasticity. This stochasticity affects the strength of the correlation between cues and stressors, shaping the degree of statistical predictability or intrinsic predictability of the plant's environment. In addition to stochastic processes, a discrepancy in plant responses and anticipated conditions can be caused by the delayed rate of change in communities of antagonists in response to changes in the local environment [20]. Such mismatches are promoted by the persistence of relatively long-lived individuals, the absence of better matching species in the local species pool, or due to priority effects in the assembly of communities [46].

Together with physiological and developmental constraints, and incomplete or unreliable information when mounting responses, stochastic processes and community lags limit plants in how well and for which timespan they can anticipate and match plastic responses to stress. While physiological and developmental constraints determine the minimal timespan needed to form anticipatory plastic responses, stochastic processes and incomplete information determine the timespan for which predictions are reliable (Figure 1). Finally, specific plant responses can be the result of targeted manipulation of plant metabolism by herbivores, with gall forming herbivores as a prominent case [37]. Nongalling herbivores can also manipulate plant metabolism. For example, Colorado potato beetles Leptinotarsa decemlineata contain bacteria in their oral secretions which cause tomato plants, Solanum lycopersicum, to greatly increase levels of SA, which downregulate JA-dependent defences required for resistance against the beetles [47]. This suggests that plants identify the stress as related to pathogens rather than related to stress by chewing herbivores. Hence, biotic interactions can cause plants to mismatch their responses to stress [48].

\section{Predictability of Biotic Stress Is Integrated in Plant Strategies}

Despite the physiological constraints and ecological stochasticity, there is substantial evidence that plants do integrate predictability in their strategies. The nature of these strategies is determined by the accuracy and timespan of the forecast horizon. We may expect that specific anticipatory strategies that are commonly found across populations of a plant species match processes that act on large spatial or temporal scales such as climatic conditions or migration patterns of herbivores [49,50]. More uncommon or plant-population-specific anticipatory strategies include adaptations to local dynamics in herbivore communities.

\section{Ontogenetic Trajectories}

When probability and patterns of herbivore attack change in a predictable way throughout the lifetime of plants, defensive traits can be expressed through fixed ontogenetic trajectories which allow the optimisation of resources and functions across plant development [15]. These trajectories can have significant genetic variation and/or be phenotypically variable within populations, influenced by plastic responses to different stressors. Thus, natural selection should favour plants allocating resources to specific defensive traits only when most needed or when other functions with a greater impact on fitness are not compromised $[51,52]$. The genes underlying these allocation trade-offs, such as those between defence and growth, are now being identified [53,54].

Ontogenetic changes in resistance and tolerance are found in systems in which interactions with herbivores affecting plant fitness are relatively predictable [53]. For example, once boreal plants outgrow the height browsed by mammals, they reduce their investment in phenolic compounds, a natural deterrent against these herbivores [55]. Analogous to ontogenetic expression of direct defences, it is common to see dynamics in indirect defences that enhance herbivore predation 
by their natural enemies. Plants tend to increase domatia and extrafloral nectaries as plants develop [56]. This increased emphasis on indirect defences during ontogeny could be driven by the likelihood of encountering natural enemies that are foraging for plant rewards, the resources available to produce such traits, mutualism management strategies, and/or tradeoffs between processes regulating reproduction and direct plant defences $[57,58]$. The adaptive value of ontogenetic changes in defence strategies matches patterns that act on longer-term scales of development (i.e. weeks to months for annuals, years for perennials) [59].

\section{Steering Development of Communities}

On shorter time scales, priming and induced responses can allow plants to cope with low predictability of herbivory or allow them to influence the sequence in which different types of herbivores occur on the plant. Induced responses to biotic and abiotic stress frequently involve fundamental changes in both primary and secondary metabolism and alter the plant as an environment for current and subsequent attackers [60,61]. Hence, the pool of herbivore species that interact with the plant after the first attacker is in part a function of the induced phenotype of the plant [62]. Plants in environments with multiple fitness-impacting herbivore species can thus be hypothesised to be under selection to predict and influence attacker sequences through their induced responses to minimize overall fitness impact (Figure 2). A major expectation of this hypothesis is that

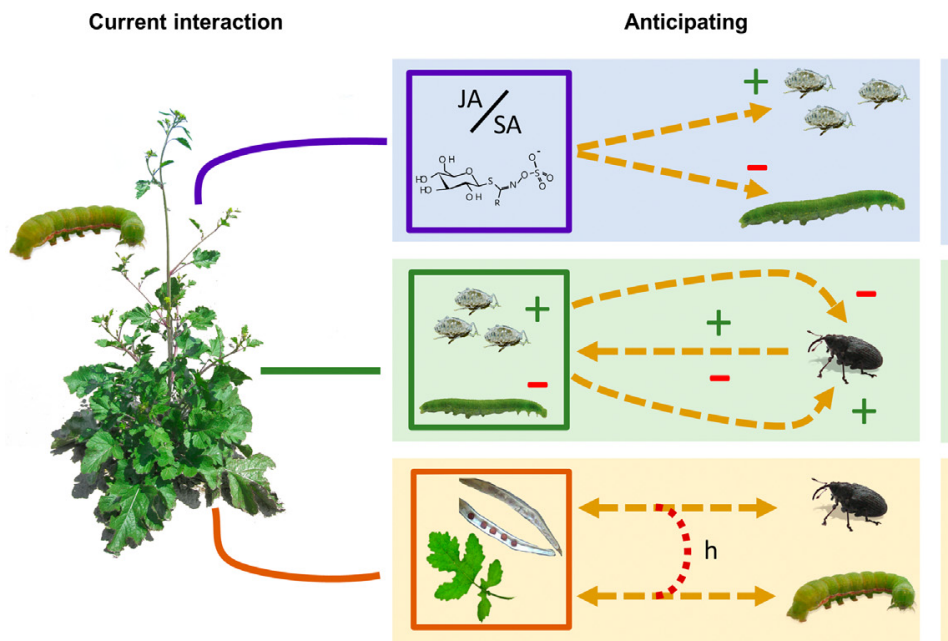

\section{Emerging property}

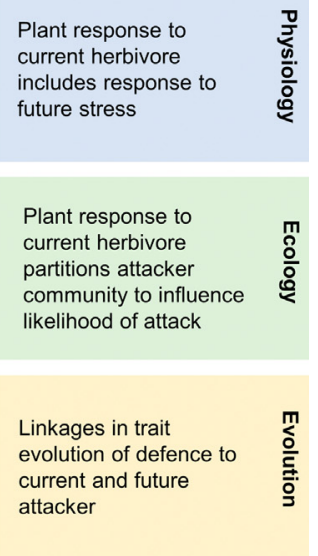

Trends in Ecology \& Evolution

Figure 2. Interactions with an Antagonist Require Anticipatory Responses to Subsequent Conditions and Results in Linkages in the Evolution of Traits. Attack by an initial stressor induces responses in the plant with physiological and ecological consequences for the plant, with the potential to ultimately change the selective pressure on plant traits. Blue: Induced responses involve (local or systemic) changes in the chemical composition of plant tissues and require the regulation of underlying phytohormonal pathways. In addition, herbivores themselves may manipulate the responses of plants on a molecular level. These changes in the plant's defensive phenotype may prove effective against subsequent attackers due to crossresistance but may also cause the plant to become more susceptible to subsequent attackers. Anticipatory responses should thus integrate, or at least not inhibit, responses to likely subsequent attack at the level of the plant's physiology. Green: Induced responses to initial stress often lead to systemic changes in the plant's phenotype. In addition, herbivores themselves may manipulate the phenotype of plants through niche construction (e.g., leafrolling caterpillars). The overall changes in the phenotype of the plant presented to the community can affect the likelihood of colonization by subsequent herbivores, effectively partitioning the antagonist community into subsets of strongly correlated antagonists. Plants may thereby anticipate the nature of future attack when interacting with the current herbivore. Orange: The interaction with an initial antagonist may result in the enhanced probability of interacting with a subsequent antagonist which has direct impacts on plant fitness. For example, a leaf feeding caterpillar enhances probability of seed weevil attack. Plant responses induced by initial herbivory may thus culminate to indirectly affect plant fitness. Plant traits that are key in mediating the initial and subsequent interactions are thus under the same selection pressure. This may result in heritability (h) of defence traits against different herbivores to be strongly linked. Abbreviations: JA: jasmonic acid; SA, salicylic acid. 
herbivore communities interacting with a plant change in their predictability after the plant interacts with antagonists. In extreme cases this could mean that plants are under selection to be attractive to herbivores that themselves have little or no significant negative fitness impact on the plant but make the plant more resistant to other, more damaging herbivores [26,63]. More generally, these ecological phenomena allow plants to fine tune their responses to current attack while optimising this response in function of the plant's community-wide context (Figure 2).

\section{Predictability in an Ecoevolutionary Context}

While predicting dynamics in stress can provide plants with a means to better match responses to the environment, it is unlikely that plants achieve a perfect prediction of future conditions given pleiotropic constraints and the lack of a guarantee of a future environment that is predicted by the past. However, fitness differences between plants that anticipate stress relative to less-informed conspecifics should be sufficient to select for plant strategies that allow for increased predictability, even if dynamics in stress are uncertain. As individual plants rarely interact with all potential antagonists in the local environment, plants may reduce their responses to only the more likely and most severe fitness-limiting stressors and anticipate the most predictable responses of other community members to the induced plant phenotype. Variation in predictability of herbivore assembly on individual plants is likely to drive the evolution of (herbivore-specific) induced defences. This may include maintenance of genetic variation in plant populations by frequencydependent selection in which genotypes are selected to optimise resistance strategies to subsets of the community consisting of strongly correlated antagonists [64]. An emerging evolutionary consequence may be that heritability of traits in resistance to different herbivores becomes linked. For example, correlation between leaf chewing herbivore attack and subsequent arrival of seed predators may yield induced responses to leaf chewers that include changes in traits of reproductive organs [63].

Rather than accurately predicting arrival of individual stressors, it is likely that plants optimise a more general cost-benefit balance to deal with multiple stressors, while managing risk by making adaptive errors under conditions of uncertain but, if realised, costly attack [22]. This suggests that a plant strategy that maximises the predictability of individual stressors is not adaptive by default: a developmental trajectory associated with higher levels of uncertainty will be selected over a developmental trajectory with predictable dynamics if the latter is associated with a lower overall fitness (Figure 1). Nevertheless, the predictability of stressors could help plants to anticipate future dynamics in costs, benefits, and risks and ultimately optimise life-history strategies [65]. The readiness of plants to respond to stimuli is likely to be dynamic over plant ontogeny, as some plant life-stages may be more vulnerable to specific stressors than other stages [66].

Finally, high levels of gene flow may prevent plants from incorporating patterns that are predictable at the local scale due to trait mismatching. However, the lack of sustained directional change towards integrating predictability in plant strategies is not indicative of the absence of selection. For example, alternative strategies can evolve within populations through balancing selection. Conversely, while we observe that many mechanisms can increase the predictability of stress in the plants' environment, not all traits involved are necessarily shaped by processes where predictability is the selective agent. It remains challenging to disentangle selective agents, as phenotypes that are selected by other stressors are likely to overlap with changes in the overall predictability of future conditions plants experience. For example, drought stress can alter plant phenotypes in ways that affect their subsequent interactions with herbivores. Even though the expressed phenotype under drought stress may increase the predictability of, and resistance against future biotic stress, it is unlikely that the induced phenotype is selected to increase predictability. 


\section{Concluding Remarks}

Anticipatory responses are ubiquitous in plants, demonstrating that the integration of correlations between stimuli and stressors in plant strategies can provide fitness benefits relative to plants with uninformed strategies [17]. Over recent years there has been a growing interest in the role such correlations play across larger (transgenerational) scales in modulating the plasticity of plant development and responses to stress, which itself may evolve $[67,68]$. However, a continuing challenge is to identify the ecological conditions under which such strategies are adaptive, and how variation in the predictability of the environment in which plants grow affects intraspecific variation in plant strategies (see Outstanding Questions) [69]. We propose that, even if dynamics in stress for the greater part remain uncertain, predictable patterns are readily integrated in plant strategies. Hence, the predictability of (sequences of) stressors is likely to be a key component governing inter- and intraspecific variation in plant responses and strategies maximising life-time fitness [64]. While studying plant interactions with stressors in isolation provides fundamental insights in the mechanisms underlying plant responses to stress, integrating predictability to the framework of plant-stressor interactions will yield important new insights in the evolution of plant defence strategies.

\section{Acknowledgements}

D.M. and E.H.P. are funded by the European Research Council (ERC) under the EU Horizon 2020 Research and Innovation Program (grant agreement no. 677139 to E.H.P.). N.K.W. is supported by the National Institute of General Medical Sciences of the National Institutes of Health (grant no. R35GM119816). We thank the New Phytologist Trust for supporting our participation to the $7^{\text {th }}$ New Phytologist Workshop: Frontiers in Chemical Ecology and Coevolution in 2013 that inspired us to collaborate on this review. We thank Marcel Dicke and three anonymous reviewers for providing feedback on an earlier version of this manuscript.

\section{References}

1. Hilker, M. and Fatouros, N.E. (2016) Resisting the onset of herbivore attack: plants perceive and respond to insect eggs. Curr. Opin. Plant Biol. 32, 9-16

2. Budaev, S. et al. (2019) Decision-making from the animal perspective: bridging ecology and subjective cognition. Front Ecol.Evol 7, 164

3. Zu, P. et al. (2020) Information arms race explains plantherbivore chemical communication in ecological communities. Science 368, 1377-1381

4. Stam, J.M. et al. (2014) Plant interactions with multiple insect herbivores: from community to genes. Annu. Rev. Plant Biol. $65,689-713$

5. Suzuki, N. et al. (2014) Abiotic and biotic stress combinations. New Phytol. 203, 32-43

6. Schuman, M.C. and Baldwin, I.T. (2016) The layers of plant responses to insect herbivores. Annu. Rev. Entomol. 61, 373-394

7. Atkinson, N.J. and Urwin, P.E. (2012) The interaction of plant biotic and abiotic stresses: From genes to the field. J. Exp. Bot. 63, 3523-3543

8. Karban, R. (2019) The ecology and evolution of induced responses to herbivory and how plants perceive risk. Ecol. Entomol. 45, 1-9

9. Nguyen, D. et al. (2016) How plants handle multiple stresses: hormonal interactions underlying responses to abiotic stress and insect herbivory. Plant Mol. Biol. 91, 727-740

10. Kroes, A et al. (2017) Brevicoryne brassicae aphids interfere with transcriptome responses of Arabidopsis thaliana to feed ing by Plutella xylostella caterpillars in a density-dependent manner. Oecologia 183, 107-120

11. Moreira, X. et al. (2018) Interactions between plant defence signalling pathways: Evidence from bioassays with insect herbivores and plant pathogens. J. Ecol. 106, 2353-2364

12. Lill, J.T. and Marquis, R.J. (2003) Ecosystem engineering by caterpillars increases insect herbivore diversity on white oak. Ecology 84, 682-690
13. Stam, J.M. et al. (2018) Order of herbivore arrival on wild cabbage populations influences subsequent arthropod community development Oikos 127, 1482-1493

14. Yang, L.H. and Cenzer, M.L. (2020) Seasonal windows of opportunity in milkweed-monarch interactions. Ecology 101 e02880

15. Barton, K.E. and Koricheva, J. (2010) The ontogeny of plant defense and herbivory: Characterizing general patterns using meta-analysis. Am. Nat. 175, 481-493

16. Humphrey, P.T. et al. (2014) Diversity and abundance of phyllosphere bacteria are linked to insect herbivory. Mol. Ecol. 23, 1497-1515

17. Karban, R. (2015) Plant Sensing and Communication, University of Chicago Press

18. Draghi, J. (2019) Phenotypic variability can promote the evolution of adaptive plasticity by reducing the stringency of natural selection. J. Evol. Biol. 32, 1274-1289

19. de Vries, J. et al. (2017) Dynamic plant-plant-herbivore interactions govern plant growth-defence integration. Trends Plant Sci. 22, 329-337

20. Blonder, B. et al. (2017) Predictability in community dynamics. Ecol. Lett. 20, 293-306

21. Petchey, O.L. et al. (2015) The ecological forecast horizon, and examples of its uses and determinants. Ecol. Lett. 18, 597-611

22. Orrock, J.L. et al. (2015) Error management in plant allocation to herbivore defense. Trends Ecol. Evol. 30, 441-445

23. Tufto, J. (2000) The evolution of plasticity and nonplastic spatia and temporal adaptations in the presence of imperfect environmental cues. Am. Nat. 156, 121-130

24. Atamian, H.S. et al. (2016) Circadian regulation of sunflower heliotropism, floral orientation, and pollinator visits. Science 353, 587-590

25. Mescher, M.C and De Moraes, C.M. (2015) Role of plant sensory perception in plant-animal interactions. J. Exp. Bot. 66, 425-433

26. Poelman, E.H. and Kessler, A. (2016) Keystone herbivores and the evolution of plant defenses. Trends Plant Sci. 21, 477-485

\section{Outstanding Questions}

What characteristics of ecological systems underly variation in the intrinsic predictability of stress and how does this affect plant life-history strategies?

What are the plant traits that perceive and process predictive environmental cues?

How plastic are plants in their sensitivity to cues and what is their tendency to take risks? Do plants exposed to a more predictable antagonist community anticipate arrival of new stressors in their plastic response to current stress, and do plants in unpredictable environments respond to each stressor in isolation when it arrives?

Does selection by a local predictable order of stressors lead to rapid evolution in plant populations in which plant traits become linked to maximise resistance to multiple stressors? 
27. Mauck, K.E. et al. (2016) Effects of pathogens on sensorymediated interactions between plants and insect vectors. Curr. Opin. Plant Biol. 32, 53-61

28. Stam, J.M. et al. (2019) Cross-seasonal legacy effects of arthropod community on plant fitness in perennial plants. J. Ecol. 107, 2451-2463

29. Toftegaard, T. et al. (2019) Butterfly-host plant synchrony determines patterns of host use across years and regions. Oikos 128, 493-502

30. Yip, E.C. et al. (2019) Trade-offs between defenses against herbivores in goldenrod (Solidago altissima). Arthropod Plant Inte. 13, 279-287

31. Quintero, C. and Bowers, M.D. (2018) Plant and herbivore ontogeny interact to shape the preference, performance and chemical defense of a specialist herbivore. Oecologia 187. 401-412

32. Cohen, D. (1967) Optimizing reproduction in a randomly varying environment when a correlation may exist between the conditions at the time a choice has to be made and the subsequent outcome. J. Theor. Biol. 16, 1-14

33. Herman, J.J. et al. (2012) Adaptive transgenerational plasticity in an annual plant: grandparental and parental drought stress enhance performance of seedlings in dry soil. Integr. Comp. Biol. 52, 77-88

34. Richards, C.L. et al. (2017) Ecological plant epigenetics: evidence from model and non-model species, and the way forward. Ecol. Lett. 20, 1576-1590

35. Futuyma, D.J. (2017) Evolutionary biology today and the call for an extended synthesis. Interface Focus 7, 20160145

36. Haselton, M.G. and Nettle, D. (2006) The paranoid optimist: an integrative evolutionary model of cognitive biases. Personal. Soc. Psychol. Rev. 10, 47-66

37. Karban, R and Orrock, J.L. (2018) A judgment and decisionmaking model for plant behavior. Ecology 99, 1909-1919

38. Abramson, C.I. and Chicas-Mosier, A.M. (2016) Learning in plants: lessons from Mimosa pudica. Front. Psychol. 7, 417

39. Donelan, S.C. et al. (2020) Transgenerational plasticity in human-altered environments. Trends Ecol. Evol. 35, 115-124

40. Karban, R. and Nagasaka, K. (2004) Are defenses of wild radish populations well matched with variability and predictablity of herbivory? Evol. Ecol. 18, 283-301

41. Maffei, M.E. et al. (2007) Before gene expression: early events in plant-insect interaction. Trends Plant Sci. 12, 310-316

42. Santamaria, M.E. et al. (2018) Plant perception and short-term responses to phytophagous insects and mites. Int. J. Mol. Sci. 19, 1356

43. Thaler, J.S. et al. (2002) Cross-talk between jasmonate and salicylate plant defense pathways: effects on several plant parasites. Oecologia 131, 227-235

44. Erb, M. et al. (2012) Role of phytohormones in insect-specific plant reactions. Trends Plant Sci. 17, 250-259

45. Weinig, C. (2000) Limits to adaptive plasticity: temperature and photoperiod influence shade-avoidance responses. Am. J. Bot. 87, 1660-1668

46. Fukami, T. et al. (2010) Assembly history dictates ecosystem functioning: evidence from wood decomposer communities. Ecol. Lett. 13, 675-684

47. Chung, S.H. et al. (2013) Colorado potato beetle manipulates plant defenses in local and systemic leaves. Plant Signal. Behav. 8, e27592

48. Humphrey, P.T. and Whiteman, N.K. (2020) Insect herbivory reshapes a native leaf microbiome. Nat. Ecol. Evol. 4, 221-229

49. Novak, M et al. (2011) Predicting community responses to perturbations in the face of imperfect knowledge and network complexity. Ecology 92, 836-846

50. Start, D. et al. (2019) Indirect interactions shape selection in a multispecies food web. Am. Nat. 193, 321-330

51. Cope, O.L. et al. (2019) Chemical defense over decadal scales: ontogenetic allocation trajectories and consequences for fitness in a foundation tree species. Funct. Ecol. 33, 2105-2115

52. Rusman, Q. et al. (2019) Ecology of plastic flowers. Trends Plant Sci. 24, 725-740

53. Ochoa-Lopez, S. et al. (2020) Ontogenetic changes in the targets of natural selection in three plant defenses. New Phytol. 226, 1480-1491
54. Leichty, A.R. and Poethig, R.S. (2019) Development and evolution of age-dependent defenses in ant-acacias. Proc. Nat. Acad. Sci. U. S. A. 116, 15596-15601

55. Bryant, J.P. and Julkunen-Tiitto, R. (1995) Ontogenic development of chemical defense by seedling resin birch: energy cost of defense production. J. Chem. Ecol. 21, 883-896

56. Van Wyk, J.I. et al. (2019) Plants trap pollen to feed predatory arthropods as an indirect resistance against herbivory. Ecology 100, e02867

57. Jacobsen, D.J. and Raguso, R.A. (2018) Lingering effects of herbivory and plant defenses on pollinators. Curr. Biol. 28 R1164-R1169

58. Fonseca-Romero, M.A. et al. (2019) Ontogenetic traiectories of direct and indirect defenses of myrmecophytic plants colonized either by mutualistic or opportunistic ant species. Oecologia 190, 857-865

59. Moreira, X. et al. (2020) Ontogenetic consistency in oak defence syndromes. J. Ecol. 108, 1822-1834

60. Eisenring, M. et al. (2018) Differential impact of herbivores from three feeding guilds on systemic secondary metabolite induction, phytohormone levels and plant-mediated herbivore interactions. J. Chem. Ecol. 44, 1178-1189

61. Zhou, S, et al. (2015) Alteration of plant primary metabolism in response to insect herbivory. Plant Physiol. 169, 1488-1498

62. Huang, W. et al. (2017) A mechanism for sequence specificity in plant-mediated interactions between herbivores. New Phytol. 214, 169-179

63. McArt, S.H. et al. (2013) Leaf herbivory increases plant fitness via induced resistance to seed predators. Ecology 94 966-975

64. March-Salas, M. et al. (2020) Effects of intrinsic environmenta predictability on intra-individual and intra-population variability of plant reproductive traits and eco-evolutionary consequences. Ann Bot. Published online May 18, 2020. https://doi.org/ 10.1093/aob/mcaa096

65. Dener, E. et al. (2016) Pea plants show risk sensitivity. Curr. Biol. 26, 1763-1767

66. Tsunoda, T. et al. (2017) Root and shoot glucosinolate allocation patterns follow optimal defence allocation theory. J. Ecol. 105, 1256-1266

67. Barton, K.E. and Boege, K. (2017) Future directions in the ontogeny of plant defence: Understanding the evolutionary causes and consequences. Ecol. Lett. 20, 403-411

68. Fernández-Pascual, E. et al. (2019) Seeds of future past: climate change and the thermal memory of plant reproductive traits. Biol. Rev. 94, 439-456

69. Lampei, C. (2019) Multiple simultaneous treatments change plant response from adaptive parental effects to within generation plasticity in Arabidopsis thaliana. Oikos 128, 368-379

70. Whitfeld, T.J.S. et al. (2012) Predicting tropical insect herbivore abundance from host plant traits and phylogeny. Ecology 93, S211-S222

71. Visakorpi, K. et al. (2019) Insect community structure covaries with host plant chemistry but is not affected by prior herbivory. Ecology 100, e02739

72. Andrews, E.S. et al. (2007) Pollinator and herbivore attraction to Cucurbita floral volatiles. J. Chem. Ecol. 33, 1682-1691

73. He, H. et al. (2016) Effects of parental temperature and nitrate on seed performance are reflected by partly overlapping genetic and metabolic pathways. Plant Cell Physiol. 57, 473-487

74. Singh, P. et al. (2017) Jasmonic acid-dependent regulation of seed dormancy following maternal herbivory in Arabidopsis. New Phytol. 214, 1702-1711

75. Baker, B.H. et al. (2019) Transgenerational effects of parental light environment on progeny competitive performance and lifetime fitness. Philos. Trans. R. Soc. Lond. B. Biol. Sci. 374, 20180182

76. Liu, Y. et al. (2018) Light quality affects flavonoid production and related gene expression in Cyclocarya paliurus. J. Photochem. Photobiol. B 179, 66-73

77. Nitschke, S. et al. (2017) Novel stress in plants by altering the photoperiod. Trends Plant Sci. 22, 913-916

78. Yoshida, T. et al. (2011) Arabidopsis HsfA1 transcription factors function as the main positive regulators in heat shock- 
responsive gene expression. Mol. Gen. Genomics. 286, 321-332

79. Kidokoro, S. et al. (2017) Different cold-signaling pathways function in the responses to rapid and gradual decreases in temperature. Plant Cell 29, 760-774

80. Hodges, J.A. et al. (2019) Evidence for direct effects of firecues on germination of some perennial forbs common in grassy ecosystems. Austral. Ecol. 44, 1271-1284

81. Pavlovic, L et al. (2019) Early brassica crops responses to salinity stress: a comparative analysis between chinese cabbage, white cabbage, and kale. Front. Plant Sci. 10, 450

82. Falik, O. et al. (2005) Root navigation by self inhibition. Plant Cell Environ. 28, 562-569

83. Ghosh, R. et al. (2017) Expression analysis of sound vibrationregulated genes by touch treatment in Arabidopsis. Front. Plant Sci. 8, 100

84. Smith, V.C. and Ennos, A.R. (2003) The effects of air flow and stem flexure on the mechanical and hydraulic properties of the stems of sunflowers Helianthus annuus. J. Exp. Bot. 54, 845-849

85. Okamoto, K. et al. (2015) Regulation of organ straightening and plant posture by an actin-myosin XI cytoskeleton. Nat. Plants 1, 1-7

86. Takahashi, N. et al. (2002) Hydrotropism in abscisic acid, wavy, and gravitropic mutants of Arabidopsis thaliana. Planta 216, 203-211

87. Duque, L et al. (2019) Plant-mediated effects of ozone on herbivores depend on exposure duration and temperature. Sci. Rep. 9, 1-11

88. Bargmann, T. et al. (2014) Life after fire: smoke and ash as germination cues in ericads, herbs and graminoids of northern heathlands. Appl. Veg. Sci. 17, 670-679

89. Svistoonoff, S. et al. (2007) Root tip contact with lowphosphate media reprograms plant root architecture. Nat. Genet. 39, 792-796

90. Babikova, Z. et al. (2013) Underground signals carried through common mycelial networks warn neighbouring plants of aphid attack. Ecol. Lett. 16, 835-843

91. Veits, M. et al. (2019) Flowers respond to pollinator sound within minutes by increasing nectar sugar concentration. Ecol. Lett. 22, 1483-1492

92. Karban, R. et al. (2014) Volatile communication between plants that affects herbivory: A meta-analysis. Ecol. Lett. 17, 44-52

93. Runyon, J.B et al (2006) Volatile chemical cues quide host location and host selection by parasitic plants. Science 313, 1964-1967
94. Kong, C.H. et al. (2018) Plant neighbor detection and allelochemical response are driven by root-secreted signaling chemicals. Nat. Commun. 9, 3867

95. Yoshinaga, N. et al. (2014) N-(18-Hydroxylinolenoyl)-L-glutamine: a newly discovered analog of volicitin in Manduca sexta and its elicitor activity in plants. J. Chem. Ecol. 40, 484-490

96. Appel, H.M. and Cocroft, R.B. (2014) Plants respond to leaf vibrations caused by insect herbivore chewing. Oecologia $175,1257-1266$

97. Balbyshev, N.F. and Lorenzen, J.H. (1997) Hypersensitivity and egg drop: a novel mechanism of host plant resistance to colorado potato beetle (Coleoptera: Chrysomelidae). J. Econ Entomol. 90, 652-657

98. Fatouros, N.E. et al. (2005) Oviposition-induced plant cues: do they arrest trichogramma wasps during host location? Entomol. Exp. Appl. 115, 207-215

99. Ray, S. et al. (2015) Maize plants recognize herbivoreassociated cues from caterpillar frass. J. Chem. Ecol. 41 781-792

100. Schwartzberg, E.G. and Tumlinson, J.H. (2014) Aphid honeydew alters plant defence responses. Funct. Ecol. 28, 386-394

101. Ingwell, L.L. et al. (2012) Plant viruses alter insect behavior to enhance their spread. Sci. Rep. 2, 578

102. Sarkar, N. et al. (2016) Volatiles of Solena amplexicaulis (lam.) gandhi leaves influencing attraction of two generalist insect herbivores. J. Chem. Ecol. 42, 1004-1015

103. Agrawal, A.A. (2000) Specificity of induced resistance in wild radish: causes and consequences for two specialist and two generalist caterpillars. Oikos 89, 493-500

104. Feng, S.H. et al. (2010) Epigenetic reprogramming in plant and animal development. Science 330, 622-627

105. Rasmann, S. et al. (2012) Herbivory in the previous generation primes plants for enhanced insect resistance. Plant Physiol. $158,854-863$

106. Colicchio, J. (2017) Transgenerational effects alter plant defence and resistance in nature. J. Evol. Biol. 30, 664-680

107. Prizak, R. et al. (2014) Fitness consequences of maternal and grandmaternal effects. Ecol. Evol. 4, 3139-3145

108. Ballhorn, D.J. et al. (2016) Herbivore damage induces a transgenerational increase of cyanogenesis in wild lima bean (Phaseolus lunatus). Chemoecology 26, 1-5

109. Pashalidou, F.G. et al. (2020) Plant volatiles induced by herbivore eggs prime defences and mediate shifts in the reproductive strategy of receiving plants. Ecol. Lett. 23, $1097-1106$ 